\title{
Improved photoluminescence of InGaAsN-(In)GaAsP quantum well by organometallic vapor phase epitaxy using growth pause annealing
}

\author{
Nelson Tansu, ${ }^{\text {a) }}$ Jeng-Ya Yeh, and Luke J. Mawst \\ Reed Center for Photonics, Department of Electrical Computer Engineering, \\ University of Wisconsin-Madison, 1415 Engineering Drive, Madison, Wisconsin 53706-1691
}

(Received 4 November 2002; accepted 11 March 2003)

\begin{abstract}
The metalorganic chemical vapor deposition of a highly strained InGaAsN quantum-well (QW) surrounded by (In)GaAsP direct barrier layers is investigated. We found that growth pause annealing with $\mathrm{AsH}_{3}$, performed immediately before and after the growth of the $\mathrm{QW}$, significantly improves the optical quality of InGaAsN QW with (In)GaAsP direct barriers. The utilization of larger band gap barrier materials, such as InGaAsP or GaAsP, will potentially lead to reduced carrier leakage from the QW laser structures. (C) 2003 American Institute of Physics. [DOI: 10.1063/1.1572470]
\end{abstract}

The InGaAsN quantum-well (QW) active region on GaAs has demonstrated excellent lasing characteristics for high-performance $1300 \mathrm{~nm}$ semiconductor lasers, ${ }^{1-11}$ comparable to or superior to some of the best published results based on the conventional InP technology. ${ }^{12,13}$ Unfortunately, high-performance $1300 \mathrm{~nm}$ InGaAsN QW lasers exhibit low characteristic temperature coefficients $\left(1 / T_{0}=(1 / J\right.$ th $) d J$ th/ $d T$ ) of the threshold-current density ( $J$ th), with only a slight improvement in $T_{0}$ values over those achievable by the conventional InP technology. Some recent works ${ }^{14}$ have attributed the poor $T_{0}$ values in $1300 \mathrm{~nm}$ InGaAsN QW lasers to the existence of large Auger recombination processes. Other recent work ${ }^{15}$ has identified that increased carrier leakage processes and a more temperature sensitive material gain parameter play a pivotal role in leading to increased temperature sensitivity of $1300 \mathrm{~nm}$ InGaAsN QW lasers.

The motivation of this work was to investigate the metalorganic chemical vapor deposition (MOCVD) growth of InGaAsN QWs with various larger band gap barrier materials. All previous studies of MOCVD-grown InGaAsN QWs have pursued GaAs as the direct barrier material. ${ }^{1-10}$ There have been several works investigating the growth of InGaAsN QWs with GaAsN as direct barriers, ${ }^{4,7,8}$ which however, is a smaller band gap material system. The utilization of larger band gap barrier materials will potentially lead to the suppression of thermionic carrier leakage, ${ }^{16}$ which will in turn lead to a reduction in the temperature sensitivity of the threshold-current density of the lasers, in particular, at hightemperature operation. The choices of larger band gap materials employed in these studies include GaAsP and InGaAsP material systems.

All structures reported here were grown by utilizing lowpressure MOCVD. The group-V precursors are the hydride sources of $\mathrm{AsH}_{3}$ and $\mathrm{PH}_{3}$. The trimethyl precursors of gallium $(\mathrm{Ga})$, aluminum (Al), and indium (In) are the group-III precursors. The $\mathrm{N}$ precursor is $\mathrm{U}$-dimethylhydrazine. The active regions are all based on the $60 \AA \mathrm{In}_{0.4} \mathrm{Ga}_{0.6} \mathrm{As}_{0.995} \mathrm{~N}_{0.005}$ $\mathrm{QW}$, sandwiched with various barrier materials. The In and $\mathrm{N}$ contents of the InGaAsN material were determined from

${ }^{a)}$ Electronic mail: tansu@cae.wisc.edu high-resolution x-ray diffraction and secondary mass ion spectroscopy measurements.

A schematic diagram of the structure investigated here is shown in Fig. 1. The experiments are conducted for a InGaAsN single-QW active region with GaAs, GaAsP, and InGaAsP direct barriers. The lower cladding layer of the structure consists of $0.1 \mu \mathrm{m} n-\mathrm{Al}_{0.74} \mathrm{Ga}_{0.26} \mathrm{As}$, grown at a temperature of $775^{\circ} \mathrm{C}$. The active region and the direct barrier materials are sandwiched in a $3000 \AA$ thick GaAs optical confinement region. The active region, barriers, and the optical confinement regions are all grown at a temperature of $530^{\circ} \mathrm{C}$. The thermal annealing of the active region occurs during the growth of the top cladding layer of $0.1 \mu \mathrm{m}$ $p$ - $\mathrm{Al}_{0.74} \mathrm{Ga}_{0.26}$ As at a temperature of $700^{\circ} \mathrm{C}$ for a duration of approximately $3 \mathrm{~min}$. The active region, barrier region, and optical confinement regions are not intentionally doped, and the doping level of both cladding layers is approximately 1 $\times 10^{18} \mathrm{~cm}^{-3}$. The thermal annealing processes for all of the InGaAsN samples studied here are performed under similar conditions. Since the purpose of this experiment was to investigate the relative changes in luminescence intensity resulting from varying the MOCVD growth processes, this thermal annealing condition does not necessarily represent the most optimized condition for InGaAsN QW active regions.

The first structure investigated, as shown in Fig. 2(a), is the reference sample consisting of a InGaAsN single-QW active region with $100 \AA$ GaAs direct barriers, which also

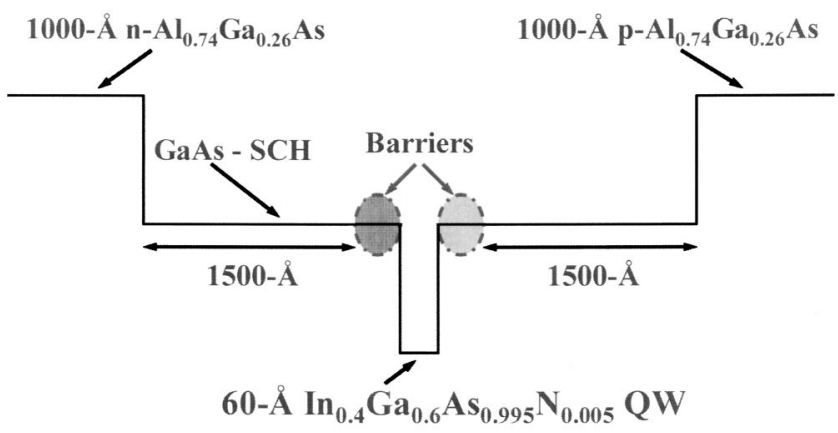

FIG. 1. The schematic layer structure of the photoluminescence sample with an $\mathrm{InGaAsN} \mathrm{QW}$ active region. 


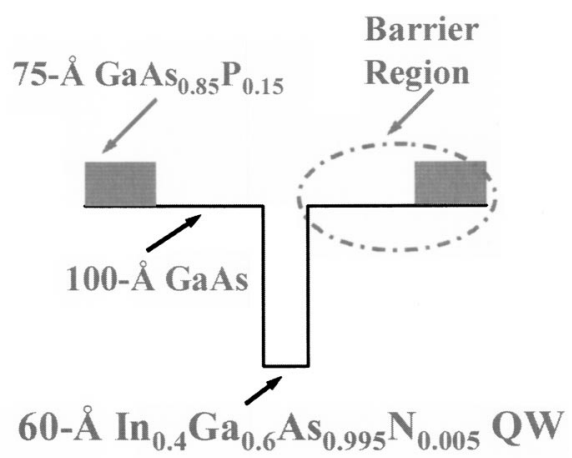

(a)

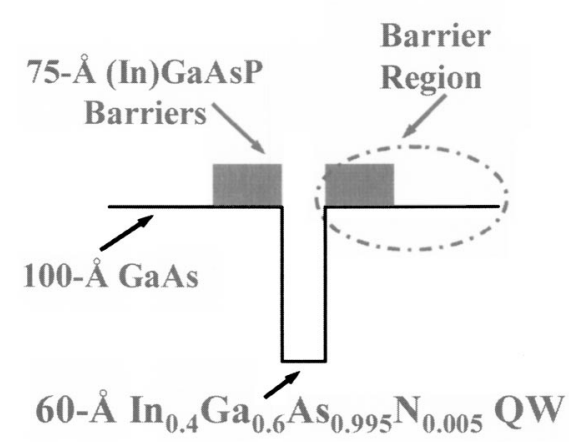

(b)

FIG. 2. The schematic diagram of the InGaAsN QW with direct barriers of (a) GaAs and (b) (In)GaAsP.

employs strain compensating layers of $75 \AA \mathrm{GaAs}_{0.85} \mathrm{P}_{0.15}$ located outside of the GaAs direct barriers to the QW. Similar active region designs have been employed for realizing high-performance $1300 \mathrm{~nm}$ InGaAsN QW lasers. ${ }^{1-3}$ The second sample studied here, shown in Fig. 2(b), consists of a InGaAsN single-QW with $75 \AA \mathrm{GaAs}_{0.85} \mathrm{P}_{0.15}$ direct barriers. The third sample consists of a InGaAsN single-QW with 75 $\AA$ InGaAsP direct barriers. The InGaAsP barrier consists of $1.62 \mathrm{eV}$ band gap quaternary material with In and P content of approximately $30 \%$ and $60 \%$, respectively, corresponding to slight tensile strain of $\Delta a / a=-1300 \mathrm{ppm}$. All of the aforementioned structures are sandwiched inside a separate confinement heterostructure consisting of $3000 \AA$ undoped GaAs and AlGaAs cladding layers, as shown in Fig. 1. Measurements of the optical luminescence are conducted by utilizing an $\mathrm{Ar}^{+}$laser with an emission wavelength of $488 \mathrm{~nm}$ and a cooled Ge detector, with all samples held at room temperature.

By incorporating an intentional growth pause annealing in the MOCVD growth processes before and after the growth of the InGaAsN QW, significant improvement in the photoluminescence is observed. The growth pause annealing is conducted by flowing $10 \mathrm{sccm}$ of $\mathrm{AsH}_{3}$ and $5200 \mathrm{sccm}$ of $\mathrm{H}_{2}$ for the pause time duration $\left(\tau_{p}\right)$. As shown in Fig. 3, the InGaAsN QW with GaAs direct barriers is shown to have luminescence intensity improvement up to two to three times by incorporating a growth pause of approximately $12 \mathrm{~s}$ before and after the growth of the QW, as shown in Fig. 3. It should be noted that high-performance $1300 \mathrm{~nm}$ InGaAsN QW lasers with GaAs barriers have also been realized previously, without the use of any growth pause. ${ }^{1-3}$

In contrast to the GaAs barrier structure, structures emIn contrast to the GaAs barrier structure, structures em-

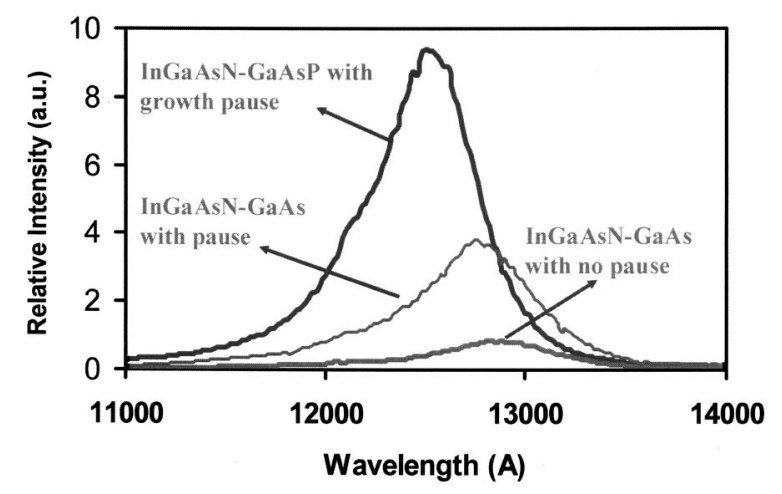

FIG. 3. The comparison of the photoluminescence spectra of $\mathrm{In}_{0.4} \mathrm{Ga}_{0.6} \mathrm{As}_{0.995} \mathrm{~N}_{0.005} \mathrm{QW}$ with GaAs and $\mathrm{GaAs}_{0.85} \mathrm{P}_{0.15}$ direct barriers, for the case of with and without pause.

ploying GaAsP or InGaAsP barriers exhibit a strong luminescence dependence on growth pause time. Without employing a growth pause before and after the InGaAsN QW, no luminescence intensity was measured from structures with direct barriers of $\mathrm{GaAs}_{0.85} \mathrm{P}_{0.15}$. By incorporating a pause of approximately 10 and $15 \mathrm{~s}$ before and after the growth of the InGaAsN QW, respectively, a significant improvement in luminescence of the InGaAsN-GaAsP structure is observed with a wavelength emission of $1.26-1.275 \mu \mathrm{m}$ as shown in Fig. 3. The shorter peak emission wavelength from the InGaAsN QW with direct barriers of GaAsP is a result of a stronger quantum confinement effect as well as growth-togrowth variation in composition.

Similar results were also found for the InGaAsNInGaAsP structures. The $1.62 \mathrm{eV}$ InGaAsP direct barriers are designed as slightly tensile strained, which provides partial strain compensation of the highly compressively strained InGaAsN QW. The pause time $\left(\tau_{p}\right)$ before and after the growth of the InGaAsN QW are kept the same for simplicity. In Fig. 4, the photoluminescence of the InGaAsN QW with InGaAsP direct barriers shows the trend of increasing luminescence intensity as the pause time is increased. The peak emission wavelengths of the InGaAsN-InGaAsP structure range from $1245 \mathrm{~nm}$ to $1252 \mathrm{~nm}$, due to run-to-run variation in MOCVD growth processes, as shown in Fig. 4. The larger quantum confinement effect (i.e., larger barrier potential) also contributes to the significantly shorter peak emission wavelength of InGaAsN-InGaAsP in comparison to the

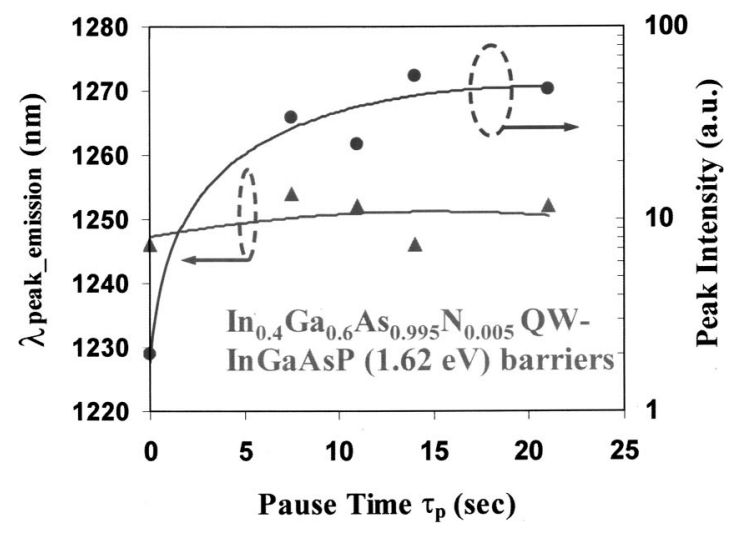

FIG. 4. The luminescence intensity and the peak emission wavelength of $\mathrm{In}_{0.4} \mathrm{Ga}_{0.6} \mathrm{As}_{0.995} \mathrm{~N}_{0.005} \mathrm{QW}$ with $1.62 \mathrm{eV}$ InGaAsP direct barriers, as a function of the pause time $\left(\tau_{p}\right)$. 


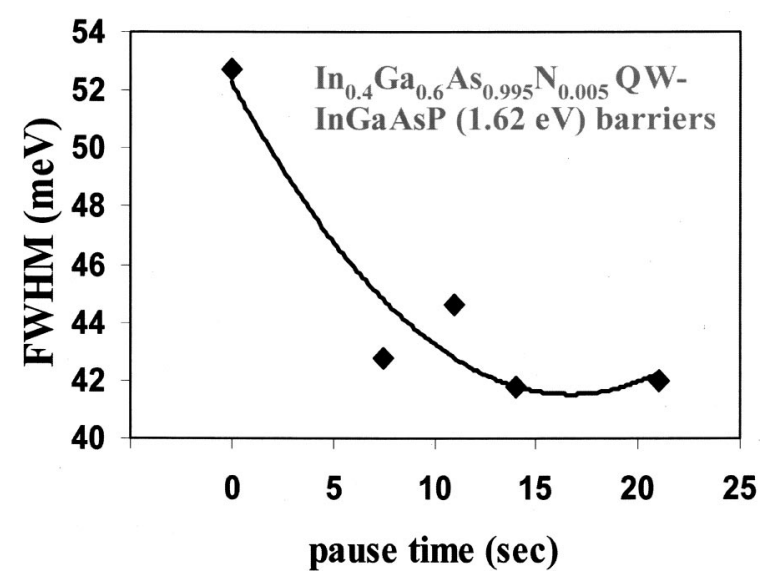

FIG. 5. The FWHM of the optical luminescence spectra for $\mathrm{In}_{0.4} \mathrm{Ga}_{0.6} \mathrm{As}_{0.995} \mathrm{~N}_{0.005} \mathrm{QW}$ with $1.62 \mathrm{eV}$ InGaAsP direct barriers, as functions of the pause time $\left(\tau_{p}\right)$.

structure with GaAs barriers. The luminescence intensity of the InGaAsN-InGaAsP structure, without employing growth pause annealing, is extremely poor, almost 1000 times smaller than that of the InGaAsN-GaAs structure without any growth pause annealing. By incorporating the growth pause annealing under $\mathrm{AsH}_{3}$ flow, significant improvement in the optical luminescence by a factor of 25 is observed, as shown in Fig. 4. This improvement is also accompanied by a reduction in the full width half maximum (FWHM) of the luminescence spectra by approximately $20 \%$, as shown in Fig. 5. It is also crucial to note that the $\mathrm{N}$-free $\mathrm{In}_{0.4} \mathrm{Ga}_{0.6} \mathrm{As}$ single-QW with either GaAs or InGaAsP direct barriers, exhibits comparable luminescence intensity without the need for a growth pause annealing.

It is important to note that the improvement in the optical luminescence of the InGaAsN QW (from the growth pause) is not accompanied by a reduction in the peak emission wavelength (Fig. 4), which indicates that the outdiffusion of $\mathrm{N}$ from QW is not the contributing factor. The growth pause annealing process of the InGaAsN QW is very different compared to the rapid thermal annealing or in situ thermal annealing process, in which the two latter processes are performed at a temperature higher than the growth temperature of the QW and are typically accompanied by a significant reduction in peak emission wavelength. Further studies indicate that the growth pause before the growth of the $\mathrm{QW}$ is most important. From the fact that no blueshift is observed with increasing annealing time, we believe that the improvement by growth pause annealing processes can possibly be attributed to an improvement in surface morphology and/or interfaces of the InGaAsN QW-(In)GaAsP direct barriers or a suppression in the $\mathrm{P}$ (phosphorous) carryover from the barriers into the QW.

In summary, we have investigated the MOCVD growth processes of strain-compensated $\mathrm{In}_{0.4} \mathrm{Ga}_{0.6} \mathrm{As}_{0.995} \mathrm{~N}_{0.005}$ QWs with larger band gap materials of $\mathrm{GaAs}_{0.85} \mathrm{P}_{0.15}$ and $1.62 \mathrm{eV}$ InGaAsP as direct barriers, utilizing $\mathrm{AsH}_{3}$ as the As precursor. From our studies, we found that the optical luminescence of the InGaAsN QW with GaAsP and InGaAsP direct barriers is significantly improved by employing a growth pause annealing with $\mathrm{AsH}_{3}$ before and after the growth of InGaAsN QW. This improvement is not followed by changes in emission wavelength, which indicates that the outdiffusion of $\mathrm{N}$ from the QW is not a factor in the improvement of optical luminescence. The utilization of larger band gap material barriers surrounding the InGaAsN QW can potentially lead to reduced thermionic carrier leakage out of the QW, which in turn will lead to a reduction in temperature sensitivity of high-performance InGaAsN QW lasers.

${ }^{1}$ N. Tansu and L. J. Mawst, IEEE Photonics Technol. Lett. 14, 444 (2002).

${ }^{2}$ N. Tansu, N. J. Kirsch, and L. J. Mawst, Appl. Phys. Lett. 81, 2523 (2002).

${ }^{3}$ N. Tansu, A. Quandt, M. Kanskar, W. Mulhearn, and L. J. Mawst (unpublished).

${ }^{4}$ D. A. Livshits, A. Y. Egorov, and H. Riechert, Electron. Lett. 36, 1381 (2000).

${ }^{5}$ T. Takeuchi, Y.-L. Chang, M. Leary, A. Tandon, H.-C. Luan, D. P. Bour, S. W. Corzine, R. Twist, and M. R. Tan, Proceedings of the IEEE LEOS 2001 Post-Deadline Session, 2001.

${ }^{6}$ M. Kawaguchi, T. Miyamoto, E. Gouardes, D. Schlenker, T. Kondo, F. Koyama, and K. Iga, Jpn. J. Appl. Phys., Part 2 40, L744 (2001).

${ }^{7}$ W. Ha, V. Gambin, M. Wistey, S. Bank, S. Kim, and J. S. Harris, Jr., IEEE Photonics Technol. Lett. 14, 591 (2002).

${ }^{8}$ C. S. Peng, T. Jouhti, P. Laukkanen, E.-M. Pavelescu, J. Konttinen, W. Li, and M. Pessa, IEEE Photonics Technol. Lett. 14, 275 (2002).

${ }^{9}$ F. Hohnsdorf, J. Koch, S. Leu, W. Stolz, B. Borchert, and M. Druminski, Electron. Lett. 35, 571 (1999).

${ }^{10}$ J. Wei, F. Xia, C. Li, and S. R. Forrest, IEEE Photonics Technol. Lett. 14, 597 (2002).

${ }^{11}$ K. D. Choquette, J. F. Klem, A. J. Fischer, O. Blum, A. A. Allerman, I. J. Fritz, S. R. Kurtz, W. G. Breiland, R. Sieg, K. M. Geib, J. W. Scott, and R. L. Naone, Electron. Lett. 36, 1388 (2000).

${ }^{12}$ P. Savolainen, M. Toivonen, P. Melanen, V. Vilokkinen, M. Saarinen, S. Orsila, T. Kuuslahti, A. Salokatve, H. Asonen, T. Panarello, R. Murison, and M. Pessa, in Proceedings of the 11th IPRM 1999, 1999, Paper MoP09.

${ }^{13}$ G. L. Belenky, C. L. Reynolds, Jr., D. V. Donetsky, G. E. Shtengel, M. S. Hybertsen, M. A. Alam, G. A. Baraff, R. K. Smith, R. F. Kazarinov, J. Winn, and L. E. Smith, IEEE J. Quantum Electron. 35, 1515 (1999).

${ }^{14}$ R. Fehse, S. Jin, S. J. Sweeney, A. R. Adams, E. P. O’Reilly, S. Illek, A. Y. Egorov, and H. Riechert, Proc. IEEE 1, 330 (2001).

${ }^{15}$ N. Tansu and L. J. Mawst, IEEE Photonics Technol. Lett. 14, 1052 (2002).

${ }^{16}$ N. Tansu and L. J. Mawst, Appl. Phys. Lett. 82, 1500 (2003). 